\author{
M. B. AHAMED
}

\title{
ON UNIQUENESS OF TWO MEROMORPHIC FUNCTIONS SHARING THREE SETS WITH FINITE WEIGHTS
}

M. B. Ahamed. On uniqueness of two meromorphic functions sharing three sets with finite weights, Mat. Stud. 53 (2020), 147-158.

With the help of the notion of weighted sharing of sets, this paper deal with the question posed by $\mathrm{Yi}[17]$ regarding the uniqueness of meromorphic functions concerning three set sharing. A result has been proved which improved the recent results of Banerjee-Ahamed [3], Banerjee-Mukherjee [5] and Banerjee-Majumder [4] by relaxing the nature of sharing. Several examples have been exhibited to show the sharpness of the cardinalities of the sets $\mathcal{S}_{1}$ and $\mathcal{S}_{2}$ considered in Theorem 1. Moreover, we give some constructive examples to endorse the validity of our established theorem.

1. Introduction, definitions and results. In this paper by a meromorphic function we will always mean a meromorphic function in the open complex plane. Let $f$ and $g$ be two non-constant meromorphic functions and let $a \in \mathbb{C} \cup\{\infty\}$. For standard definitions and notations of value distribution theory we refer to the reader to see [9]. We denote through out the paper $\mathbb{C}^{*}=\mathbb{C} \backslash\{0\}$.

If $f$ and $g$ have the same set of $a$-points with same multiplicities then we say that $f$ and $g$ share the value $a \mathrm{CM}$ (Counting Multiplicities). If we do not take the multiplicities into account, $f$ and $g$ are said to share the value $a$ IM (Ignoring Multiplicities).

Definition 1. For a non-constant meromorphic function $f$ and any set $\mathcal{S} \subset \overline{\mathbb{C}}$, we define

$$
\begin{aligned}
E_{f}(\mathcal{S})= & \bigcup_{a \in \mathcal{S}}\{(z, p) \in \mathbb{C} \times \mathbb{N}: f(z)=a \text { with multiplicity } p\}, \\
& \bar{E}_{f}(\mathcal{S})=\bigcup_{a \in \mathcal{S}}\{(z, 1) \in \mathbb{C} \times\{1\}: f(z)=a\} .
\end{aligned}
$$

If $E_{f}(\mathcal{S})=E_{g}(\mathcal{S})\left(\bar{E}_{f}(\mathcal{S})=\bar{E}_{g}(\mathcal{S})\right)$ then we simply say $f$ and $g$ share $\mathcal{S}$ Counting Multiplicities (CM) (Ignoring Multiplicities (IM)).

Evidently, if $\mathcal{S}$ contains one element only, then it coincides with the usual definition of CM (IM) sharing of values.

Next we explain some definitions and notations which will be used in the paper.

Definition 2 ([12]). Let $p$ be a positive integer and $a \in \mathbb{C} \cup\{\infty\}$.

2020 Mathematics Subject Classification: 30D35.

Keywords: meromorphic function; uniqueness; shared sets; Gross question; weighted sharing.

doi:10.30970/ms.53.2.147-158

(C) M. B. Ahamed, 2020 
(i) $N\left(r, \frac{1}{f-a} \mid \geq p\right)\left(\bar{N}\left(r, \frac{1}{f-a} \mid \geq p\right)\right)$ denotes the counting function (reduced counting function) of those $a$-points of $f$ whose multiplicities are not less than $p$.

(ii) $N\left(r, \frac{1}{f-a} \mid \leq p\right)\left(\bar{N}\left(r, \frac{1}{f-a} \mid \leq p\right)\right)$ denotes the counting function (reduced counting function) of those $a$-points of $f$ whose multiplicities are not greater than $p$.

Definition 3. Let $f$ and $g$ be two non-constant meromorphic functions such that $f$ and $g$ share the value $a$ with weight $k$ where $a \in \mathbb{C} \cup\{\infty\}$. Let $f$ and $g$ have same $a$-points with respective multiplicities $p$ and $q$. We denote by $\bar{N}_{E}^{(k+1}\left(r, \frac{1}{f-a}\right)$ the counting function of those $a$-points of $f$ and $g$ where $p=q \geq k+1$, each point in this counting function counted only once.

Definition 4 ([16]). For $a \in \mathbb{C} \cup\{\infty\}$ and a positive integer $p$ we denote by

$$
N_{p}\left(r, \frac{1}{f-a}\right)=\bar{N}\left(r, \frac{1}{f-a}\right)+\bar{N}\left(r, \frac{1}{f-a} \mid \geq 2\right)+\ldots+\bar{N}\left(r, \frac{1}{f-a} \mid \geq p\right) .
$$

It is clear that $N_{1}\left(r, \frac{1}{f-a}\right)=\bar{N}\left(r, \frac{1}{f-a}\right)$.

Definition 5. Let $N_{1)}\left(r, \frac{1}{f-a}\right)$ denote the counting function of the simple zeros of $f-a$ and $\bar{N}_{(2}\left(r, \frac{1}{f-a}\right)$ denote the reduced counting function of the $a$-points of $f$ of multiplicities $\geq 2$. It follows that

$$
N_{2}\left(r, \frac{1}{f-a}\right)=N_{1)}\left(r, \frac{1}{f-a}\right)+2 \bar{N}_{(2}\left(r, \frac{1}{f-a}\right) .
$$

Definition 6 ([19]). For a positive integer $p$ and $a \in \mathbb{C} \cup\{\infty\}$, we put

$$
\delta_{p}(a ; f)=1-\limsup _{r \longrightarrow \infty} \frac{N_{p}\left(r, \frac{1}{f-a}\right)}{T(r, f)}, \quad \Theta(a ; f)=1-\limsup _{r \longrightarrow \infty} \frac{\bar{N}\left(r, \frac{1}{f-a}\right)}{T(r, f)}
$$

Clearly $0 \leq \delta(a ; f) \leq \delta_{p}(a ; f) \leq \delta_{p-1}(a ; f) \leq \ldots \leq \delta_{2}(a ; f) \leq \delta_{1}(a ; f)=\Theta(a ; f)$.

In 1926, Nevanlinna first showed that a non-constant meromorphic function on the complex plane $\mathbb{C}$ is uniquely determined by the pre-images, ignoring multiplicities, of 5 distinct values (including infinity). A few years latter, he showed that when multiplicities are taken into consideration, 4 points are enough and in that case either the two functions coincides or one is the bilinear transformation of the other one.

The uniqueness problem for entire or meromorphic functions sharing sets was initiated by a famous question of Gross in [8]. In 1976, Gross [8] asked the following question.

Question 1. Can one find two finite sets $\mathcal{S}_{j},(j=1,2)$ such that any two non-constant entire functions $f$ and $g$ satisfying $E_{f}(\mathcal{S})=E_{g}(\mathcal{S}),(j=1,2)$ must be identical?

In [8] Gross said that if the answer of Question 1 is affirmative it would be interesting to know how large both sets would have to be?

In 1994, Yi ([17]) posed the following question.

Question 2. Can one find three finite sets $\mathcal{S}_{j},(j=1,2,3)$ such that any two non-constant meromorphic functions $f$ and $g$ satisfying $E_{f}(\mathcal{S})=E_{g}(\mathcal{S}),(j=1,2,3)$ must be identical? 
In the same paper [17], Yi answered the Question 2 affirmatively and obtained a result by showing that there exist three finite sets $\mathcal{S}_{1}$ (with 7 elements), $\mathcal{S}_{2}$ (with 2 elements) and $\mathcal{S}_{3}$ (with 1 element) such that any two non-constant meromorphic functions $f$ and $g$ satisfying $E_{f}\left(\mathcal{S}_{j}\right)=E_{g}\left(\mathcal{S}_{j}\right),(j=1,2,3)$ must be identical.

In the direction of Question 2, Fang-Xu [7] obtained the following result.

Theorem A ([7]). Let $\mathcal{S}_{1}=\{0\}, \mathcal{S}_{2}=\left\{z: z^{3}-z^{2}-1=0\right\}$ and $\mathcal{S}_{3}=\{\infty\}$. Let $f$ and $g$ be two non-constant meromorphic functions such that $\Theta(\infty ; f)>\frac{1}{2}$ and $\Theta(\infty ; g)>\frac{1}{2}$. If $E_{f}\left(\mathcal{S}_{j}\right)=E_{g}\left(\mathcal{S}_{j}\right)$, for $j=1,2,3$ then $f \equiv g$.

Dealing with the Question 2, Qiu-Fang ([15]) obtained a result with an extra supposition that the meromorphic functions $f$ and $g$ both having poles of multiplicity $\geq 2$. In the same paper they also exhibited some examples to show that the condition on the poles of $f$ and $g$ can not be removed.

In 2004, Yi-Lin ([18]) proved the following results.

Theorem B ([18]). Let $\mathcal{S}_{1}=\{0\}, \mathcal{S}_{2}=\left\{z: z^{n}+b z^{n-1}+c=0\right\}$ and $\mathcal{S}_{3}=\{\infty\}$, where $a, b$ are non-zero constants such that $z^{n}+b z^{n-1}+c=0$ has no repeated root and $n \geq 3$ is an integer. If for two non-constant meromorphic functions $f$ and $g, E_{f}\left(\mathcal{S}_{j}\right)=E_{g}\left(\mathcal{S}_{j}\right)$, for $j=1,2,3$ and $\delta_{1}(\infty ; f)>\frac{5}{6}$, then $f \equiv g$.

Theorem C ([18]). Let $\mathcal{S}_{1}=\{0\}, \mathcal{S}_{2}=\left\{z: z^{n}+b z^{n-1}+c=0\right\}$ and $\mathcal{S}_{3}=\{\infty\}$, where $a, b$ are non-zero constants such that $z^{n}+b z^{n-1}+c=0$ has no repeated root and $n \geq 4$ is an integer. If for two non-constant meromorphic functions $f$ and $g, E_{f}\left(\mathcal{S}_{j}\right)=E_{g}\left(\mathcal{S}_{j}\right)$, for $j=1,2,3$ and $\Theta(\infty ; f)>0$, then $f \equiv g$.

Progressively the research on Question 1 for meromorphic functions as well as Question 2 gained a valuable space in the literature and now a days it has increasingly become an impressive branch of the modern uniqueness theory of meromorphic functions. During the last few years a considerable amount of work has been done to explore the possible answer to Question 2 by many mathematicians.

In 2001, the introduction of the new notion of sharing which is a scaling between CM or IM, known as weighted sharing of values and sets by Lahiri $[10,11]$ further speed up the research in the direction of Question 2.

Definition 7. Let $k \in \mathbb{N} \cup\{0\} \cup\{\infty\}$. For $a \in \mathbb{C} \cup\{\infty\}$, we denote by $E_{f}(a, k)$ the set of all $a$-points of $f$, where an $a$-point of multiplicity $m$ is counted $m$ times if $m \leq k$ and $k+1$ times if $m \geq k+1$. If $E_{f}(a, k)=E_{g}(a, k)$, we say that $f$ and $g$ share the value $a$ with weight $k$.

Definition 8. Let $\mathcal{S} \subset \mathbb{C} \cup\{\infty\}$ be non-empty and $k \in \mathbb{N} \cup\{0\} \cup\{\infty\}$. We denote by $E_{f}(\mathcal{S}, k)$ the set $E_{f}(\mathcal{S}, k)=\bigcup_{a \in \mathcal{S}} E_{f}(a, k)$.

Clearly $E_{f}(\mathcal{S})=E_{f}(\mathcal{S}, \infty)$ and $\bar{E}(\mathcal{S}, k)=E_{f}(\mathcal{S}, 0)$.

With the help of wighted sharing of sets, Banerjee-Mukherjee [5] obtained the following results.

Theorem D ([5]). Let $\mathcal{S}_{1}=\{0\}, \mathcal{S}_{2}=\left\{z: z^{n}+b z^{n-1}+c=0\right\}$ and $\mathcal{S}_{3}=\{\infty\}$, where $a$, $b$ are non-zero constants such that $z^{n}+b z^{n-1}+c=0$ has no repeated root and $n \geq 3$ is an integer. If for two non-constant meromorphic functions $f$ and $g$ having no simple pole satisfying, $E_{f}\left(\mathcal{S}_{1}, 1\right)=E_{g}\left(\mathcal{S}_{1}, 1\right), E_{f}\left(\mathcal{S}_{2}, 5\right)=E_{g}\left(\mathcal{S}_{2}, 5\right)$ and $E_{f}\left(\mathcal{S}_{3}, \infty\right)=E_{g}\left(\mathcal{S}_{3}, \infty\right)$, then $f \equiv g$. 
Theorem $\mathbf{E}([5])$. Let $\mathcal{S}_{1}=\{0\}, \mathcal{S}_{2}=\left\{z: z^{n}+b z^{n-1}+c=0\right\}$ and $\mathcal{S}_{3}=\{\infty\}$, where $a, b$ are non-zero constants such that $z^{n}+b z^{n-1}+c=0$ has no repeated root and $n \geq 3$ is an integer. If for two non-constant meromorphic functions $f$ and $g$ satisfying, $E_{f}\left(\mathcal{S}_{1}, 0\right)=E_{g}\left(\mathcal{S}_{1}, 0\right)$, $E_{f}\left(\mathcal{S}_{2}, 6\right)=E_{g}\left(\mathcal{S}_{2}, 6\right), E_{f}\left(\mathcal{S}_{3}, \infty\right)=E_{g}\left(\mathcal{S}_{3}, \infty\right)$ and $\delta_{1)}(\infty ; f)+\delta_{1)}(\infty ; g)>\frac{5}{n}$, then $f \equiv g$.

Theorem $\mathbf{F}([5])$. Let $\mathcal{S}_{1}=\{0\}, \mathcal{S}_{2}=\left\{z: z^{n}+b z^{n-1}+c=0\right\}$ and $\mathcal{S}_{3}=\{\infty\}$, where $a, b$ are non-zero constants such that $z^{n}+b z^{n-1}+c=0$ has no repeated root and $n \geq 4$ is an integer. If for two non-constant meromorphic functions $f$ and $g$ satisfying, $E_{f}\left(\mathcal{S}_{1}, 0\right)=E_{g}\left(\mathcal{S}_{1}, 0\right)$, $E_{f}\left(\mathcal{S}_{2}, 6\right)=E_{g}\left(\mathcal{S}_{2}, 6\right), E_{f}\left(\mathcal{S}_{3}, 4\right)=E_{g}\left(\mathcal{S}_{3}, 4\right)$ and $\delta_{1)}(\infty ; f)+\delta_{1)}(\infty ; g)>0$, then $f \equiv g$.

Recently Banerjee-Majumder [4] obtained two results by improving some earlier results of Banerjee $[1,2]$ as follows.

Theorem G $([4])$. Let $\mathcal{S}_{1}=\{0\}, \mathcal{S}_{2}=\left\{z: z^{n}+a z^{n-1}+b=0\right\}$ and $\mathcal{S}_{3}=\{\infty\}$, where $a, b$ are non-zero constants such that $z^{n}+a z^{n-1}+b=0$ has no repeated root and $n(\geq 4)$ be an integer. If for two non-constant meromorphic functions $f$ and $g, E_{f}\left(\mathcal{S}_{1}, k_{1}\right)=E_{g}\left(\mathcal{S}_{1}, k_{1}\right)$, $E_{f}\left(\mathcal{S}_{2}, k_{2}\right)=E_{g}\left(\mathcal{S}_{2}, k_{2}\right)$ and $E_{f}\left(\mathcal{S}_{3}, k_{3}\right)=E_{g}\left(\mathcal{S}_{3}, k_{3}\right)$, where $k_{1} \geq 0, k_{2} \geq 3, k_{3} \geq 1$ are integers satisfying

$$
3 k_{1} k_{2} k_{3}>k_{2}+3 k_{1}+k_{3}-2 k_{2} k_{3}+4 \text { and } \Theta_{f}+\Theta_{g}>0,
$$

where $\Theta_{h}=\Theta(\infty ; h)+\Theta\left(\frac{a(1-n)}{n} ; h\right)$, then $f \equiv g$.

Theorem $\mathbf{H}([4])$. Let $\mathcal{S}_{1}=\{0\}, \mathcal{S}_{2}=\left\{z: z^{n}+a z^{n-1}+b=0\right\}$ and $\mathcal{S}_{3}=\{\infty\}$, where $a, b$ are non-zero constants such that $z^{n}+a z^{n-1}+b=0$ has no repeated root and $n(\geq 3)$ be an integer. If for two non-constant meromorphic functions $f$ and $g, E_{f}\left(\mathcal{S}_{1}, k_{1}\right)=E_{g}\left(\mathcal{S}_{1}, k_{1}\right)$, $E_{f}\left(\mathcal{S}_{2}, k_{2}\right)=E_{g}\left(\mathcal{S}_{2}, k_{2}\right)$ and $E_{f}\left(\mathcal{S}_{3}, k_{3}\right)=E_{g}\left(\mathcal{S}_{3}, k_{3}\right)$, where $k_{1} \geq 0, k_{2} \geq 4, k_{3} \geq 1$ are integers satisfying

$$
2 k_{1} k_{2} k_{3}>k_{2}+2 k_{1}+k_{3}-k_{2} k_{3}+3 \text { and } \Theta_{f}+\Theta_{g}>1
$$

where $\Theta_{h}=\Theta(\infty ; h)+\Theta\left(\frac{a(1-n)}{n} ; h\right)$ then $f \equiv g$.

Earlier the problem of finding the possible answer of the Question 2 was solved by Lin$\mathrm{Yi}$ [13] who answered the Question 2 by considering the sets $\mathcal{S}_{1}=\{0\}, \mathcal{S}_{2}=\left\{z: a z^{n}-n(n-\right.$ 1) $\left.z^{2}+2 n(n-2) b z=(n-1)(n-2) b^{2}\right\}$ and $\mathcal{S}_{3}=\{\infty\}$ for $n \geq 5$, where $a, b$ are constants such that $a b^{n-2} \neq 0,2$.

In [3], Banerjee-Ahamed modified the sets $\mathcal{S}_{1}, \mathcal{S}_{2}$ so that $\mathcal{S}_{1}=\{0,1\}$, and the number of elements in the new set $\mathcal{S}_{2}$ has decreased by 1 in the optimal case. Moreover the conditions on the sharing sets $\mathcal{S}_{j},(j=1,2,3)$ has also been relaxed to the conditions of sharing $\left(\mathcal{S}_{j}, k_{j}\right)$, $(j=1,2,3)$, where $\left(k_{1}, k_{2}, k_{3}\right)=(0,3,2),(0,4,1)$.

From the above discussions, we have the following notes:

Note 1. The lower bound of the cardinality of the main range set $\mathcal{S}_{2}$ is obtained so far in Theorems A, B, D, E, H and also in the result of Qiu-Fang ([15]) is 3 with the help of some extra suppositions.

Note 2. Also one may check that the optimal choice for the weights $\left(k_{1}, k_{2}, k_{3}\right)=(0,3,1)$ in Theorem G can not be considered as it violates the condition $3 k_{1} k_{2} k_{3}>k_{2}+3 k_{1}+k_{3}-$ $2 k_{2} k_{3}+4$. 
Note 3. We also see that in Theorem $\mathrm{H}$, it is not possible to consider the weights as $\left(k_{1}, k_{2}, k_{3}\right)=(0,4,1)$ and hence as $\left(k_{1}, k_{2}, k_{3}\right)=(0,3,1)$.

Based on the above observation, for the purpose of improving all the above mentioned results further, one can ask the following question.

Question 3. Can we obtain a uniqueness result corresponding to Theorems A, B, D, E, H and Qiu-Fang [15] without the help of any extra suppositions in which the lower bound of the cardinality of the main range set will be 3 ?

If the answer of the Question 3 is found to be affirmative, then one natural question is as follows.

Question 4. Is it possible to reduce further the choice of the weights $\left(k_{1}, k_{2}, k_{3}\right)$ to $(0,3,1)$ in all the above mentioned results?

Answering Questions 2, 3 and 4 affirmatively is the main motivation of writing this paper. In this paper, we have modified the sets $\mathcal{S}_{1}=\{0\}$ by $\mathcal{S}_{1}=\left\{0, \delta_{a, b}^{n}\right\}$ and $\mathcal{S}_{2}$ by an new one and obtained two results out of which the second one directly improves all the above mentioned results.

To this end, we next suppose that $\delta_{a, b}^{n}=\frac{b(1-n)}{n a}$, where $a, b \in \mathbb{C}^{*}$ and $n \geq 3$ be an integer. We consider here the $\mathcal{S}_{1}=\left\{0, \delta_{a, b}^{n}\right\}$ as the set of zeros of the derivative of the polynomial $a z^{n}+b z^{n-1}+c$.

Theorem 1. For $n \geq 3$, let $\mathcal{S}_{1}=\left\{0, \delta_{a, b}^{n}\right\}, \mathcal{S}_{2}=\left\{z: a z^{n}+b z^{n-1}+c=0\right\}$ and $\mathcal{S}_{3}=\{\infty\}$, where $a, b, c \in \mathbb{C}^{*}=\mathbb{C} \backslash\{0\}$ be so chosen that $a z^{n}+b z^{n-1}+c=0$ has no repeated root, $c \neq$ $-\frac{b}{2 n}\left(\delta_{a, b}^{n}\right)^{n-1}$. If for two non-constant meromorphic functions $f$ and $g, E_{f}\left(\mathcal{S}_{1}, 0\right)=E_{g}\left(\mathcal{S}_{1}, 0\right)$, $E_{f}\left(\mathcal{S}_{2}, n\right)=E_{f}\left(\mathcal{S}_{2}, n\right)$ and $E_{f}\left(\mathcal{S}_{3}, n-2\right)=E_{f}\left(\mathcal{S}_{3}, n-2\right)$, then $f \equiv g$.

Corollary 1. Let $\mathcal{S}_{1}=\left\{0,-\frac{2 b}{3 a}\right\}, \mathcal{S}_{2}=\left\{z: a z^{3}+b z^{2}+c=0\right\}$ and $\mathcal{S}_{3}=\{\infty\}$, where $a, b, c \in \mathbb{C}^{*}$ be so chosen that $a z^{3}+b z^{2}+c=0$ has no repeated root, $c \neq-\frac{2 b^{3}}{27 a^{2}}$. If for two non-constant meromorphic functions $f$ and $g, E_{f}\left(\mathcal{S}_{1}, 0\right)=E_{g}\left(\mathcal{S}_{1}, 0\right), E_{f}\left(\mathcal{S}_{2}, 3\right)=E_{f}\left(\mathcal{S}_{2}, 3\right)$ and $E_{f}\left(\mathcal{S}_{3}, 1\right)=E_{f}\left(\mathcal{S}_{3}, 1\right)$, then $f \equiv g$.

Remark 1 . Clearly Corollary 1 directly improves the above mentioned results as we see that the lower bound of $n$ is 3 , with the corresponding weights $\left(k_{1}, k_{2}, k_{3}\right)=(0,3,1)$.

The following example shows that the conclusions of the Theorems 1 cease to be hold if we consider $c=-\frac{b}{2 n}\left(\delta_{a, b}^{n}\right)^{n-1}$.

Example 1. Let $a=1, b=3$, then $-\frac{b}{2 n}\left(\delta_{a, b}^{n}\right)^{n-1}=-2$. Let $c=-\frac{b}{2 n}\left(\delta_{a, b}^{n}\right)^{n-1}=-2$ and $\mathcal{S}_{2}=\left\{z: z^{3}+3 z^{2}-2=0\right\}=\{-1,-1-\sqrt{3},-1-\sqrt{3}\}$ and $\mathcal{S}_{3}=\{\infty\}$. Hence we must have $\mathcal{S}_{1}=\left\{0, \delta_{a, b}^{n}\right\}=\{0,-2\}$. Let $f(z)=\phi(z)-2$ and $g(z)=-\phi(z)$, where $\phi(z)$ is a nonconstant meromorphic function. It is clear that $E_{f}\left(\mathcal{S}_{j}\right)=E_{g}\left(\mathcal{S}_{j}\right)$ for $j=1,2,3$ and hence $E_{f}\left(\mathcal{S}_{1}, 0\right)=E_{g}\left(\mathcal{S}_{1}, 0\right), E_{f}\left(\mathcal{S}_{2}, 3\right)=E_{g}\left(\mathcal{S}_{2}, 3\right)$ and $E_{f}\left(\mathcal{S}_{3}, 1\right)=E_{g}\left(\mathcal{S}_{3}, 1\right)$ but note that $f \not \equiv g$.

The next example shows the sharpness of the cardinalities of the set $\mathcal{S}_{1}$ and the main range set $\mathcal{S}_{2}$ in the Theorem 1 .

Example 2. Let $\mathcal{S}_{2}=\left\{z: a z^{2}+b z+c=0\right\}=\{\gamma, \delta\}$, where $\gamma+\delta=-\frac{b}{a}, \gamma \delta=\frac{c}{a}$, $a, b, c \in \mathbb{C}^{*}, c \neq \frac{b^{2}}{8 a}$. Hence $\mathcal{S}_{1}=\left\{-\frac{b}{2 a}\right\}=\left\{\frac{\gamma+\delta}{2}\right\}$. Let $\mathcal{S}_{3}=\{\infty\}$ and $f(z)=h(z)+\gamma+\delta$ and $g(z)=-h(z)$, where $h(z)$ is any non-constant meromorphic function. We see that all the conditions of Theorem 1 are satisfied but $f \not \equiv g$. 
The following example shows that, the condition $b \neq 0$, in Theorem 1 , can not be removed.

Example 3. Let $b=0$, then $\delta_{a, b}^{n}=0$. Thus, we get $\mathcal{S}_{1}=\{0\}$.

$$
\text { Let } \mathcal{S}_{2}=\left\{z: a z^{3}+c=0\right\}=\left\{\sqrt[3]{-\frac{c}{a}}, \sqrt[3]{-\frac{c}{a}} \omega, \sqrt[3]{-\frac{c}{a}} \omega^{2}\right\},
$$

$a, c \in \mathbb{C}^{*}$, where $\omega$ is a cube roots of unity and $\mathcal{S}_{3}=\{\infty\}$. Let $f(z)$ be a non-constant meromorphic function and $g(z)=\omega f(z)$, where $\omega$ is a non-real cube root of unity. It is clear that $E_{f}\left(\mathcal{S}_{1}, 0\right)=E_{g}\left(\mathcal{S}_{1}, 0\right), E_{f}\left(\mathcal{S}_{2}, 3\right)=E_{g}\left(\mathcal{S}_{2}, 3\right)$ and $E_{f}\left(\mathcal{S}_{3}, 1\right)=E_{g}\left(\mathcal{S}_{3}, 1\right)$ but $f \not \equiv g$.

The next two examples show that the set $\mathcal{S}_{2}$ considered in Theorem 1 can not be replaced by any arbitrary set.

Example 4. Let $\mathcal{S}_{1}=\left\{\frac{6-\sqrt{3}}{3}, \frac{6+\sqrt{3}}{3}\right\}$,

$$
\mathcal{S}_{2}=\left\{z: z^{3}-6 z^{2}+11 z-6=0\right\}=\{1,2,3\}
$$

and $\mathcal{S}_{3}=\{\infty\}$. Let $f(z)=h(z)+4$ and $g(z)=-h(z)$, where $h(z)$ is a non-constant meromorphic function. Although we se that $E_{f}\left(\mathcal{S}_{1}, 0\right)=E_{g}\left(\mathcal{S}_{1}, 0\right), E_{f}\left(\mathcal{S}_{2}, 3\right)=E_{g}\left(\mathcal{S}_{2}, 3\right)$ and $E_{f}\left(\mathcal{S}_{3}, 1\right)=E_{g}\left(\mathcal{S}_{3}, 1\right)$ but $f \not \equiv g$.

Example 5. Let $\mathcal{S}_{1}=\left\{\frac{15-\sqrt{3}}{3}, \frac{15+\sqrt{3}}{3}\right\}$,

$$
\mathcal{S}_{2}=\left\{z: z^{3}-15 z^{2}+74 z-120=0\right\}=\{4,5,6\}
$$

and $\mathcal{S}_{3}=\{\infty\}$. Let $f(z)=\phi(z)+10$ and $g(z)=-\phi(z)$, where $\phi(z)$ is a non-constant meromorphic function. Although we se that $E_{f}\left(\mathcal{S}_{1}, 0\right)=E_{g}\left(\mathcal{S}_{1}, 0\right), E_{f}\left(\mathcal{S}_{2}, 3\right)=E_{g}\left(\mathcal{S}_{2}, 3\right)$ and $E_{f}\left(\mathcal{S}_{3}, 1\right)=E_{g}\left(\mathcal{S}_{3}, 1\right)$ but $f \not \equiv g$.

Note 4. One can find many examples by considering $\mathcal{S}_{1}$ as th set of roots of the derivative of the polynomial of degree 3 whose roots formed the set $\mathcal{S}_{2}$, where $\mathcal{S}_{2}=\{m, m+1, m+2\}$, where $m \in \mathbb{N}$, and by choosing the functions $f(z)=h(z)+2(m+1)$ and $g(z)=-h(z)$, where $h(z)$ is a non-constant meromorphic function.

2. Some lemmas. In this section, we are going to discuss some lemmas which will be needed later to prove our main results. We define, for two non-constant meromorphic functions $f$ and $g$,

$$
\mathcal{F}=\frac{f^{n-1}(a f+b)}{-c}, \quad \mathcal{G}=\frac{g^{n-1}(a g+b)}{-c} .
$$

Associated to $\mathcal{F}$ and $\mathcal{G}$, we next define $\mathcal{H}$ as follows:

$$
\mathcal{H}=\left(\frac{\mathcal{F}^{\prime \prime}}{\mathcal{F}^{\prime}}-\frac{2 \mathcal{F}^{\prime}}{\mathcal{F}-1}\right)-\left(\frac{\mathcal{G}^{\prime \prime}}{\mathcal{G}^{\prime}}-\frac{2 \mathcal{G}^{\prime}}{\mathcal{G}-1}\right)
$$

and

$$
\Psi=\frac{\mathcal{F}^{\prime}}{\mathcal{F}-1}-\frac{\mathcal{G}^{\prime}}{\mathcal{G}-1}
$$


Lemma 1 ([14]). Let $h$ be a non-constant meromorphic function and let

$$
\mathcal{R}(h)=\frac{\sum_{i=1}^{n} a_{i} h^{i}}{\sum_{j=1}^{m} b_{j} h^{j}}
$$

be an irreducible rational function in $g$ with constant coefficients $\left\{a_{i}\right\},\left\{b_{j}\right\}$, where $a_{n} \neq 0$ and $b_{m} \neq 0$. Then

$$
T(r, \mathcal{R}(h))=\max \{n, m\} T(r, h)+S(r, h) .
$$

Lemma 2. Let $\mathcal{F}$ and $\mathcal{G}$ be given by (1) satisfying $E_{\mathcal{F}}(1, q)=E_{\mathcal{G}}(1, q), 0 \leq q<\infty$ with $\mathcal{H} \not \equiv 0$, then

$$
N_{E}^{1)}\left(r, \frac{1}{\mathcal{F}-1}\right)=N_{E}^{1)}\left(r, \frac{1}{\mathcal{G}-1}\right) \leq N(r, \mathcal{H})+S(r, \mathcal{F})+S(r, \mathcal{G}) .
$$

Proof. Since $E_{\mathcal{F}}(1, q)=E_{\mathcal{G}}(1, q)$. It is clear that any simple 1-point of $\mathcal{F}$ and $\mathcal{G}$ is a zero of $\mathcal{H}$. From the construction of $\mathcal{H}$, we know that $m(r, \mathcal{H})=S(r, \mathcal{F})+S(r, \mathcal{G})$. Therefore by the First Fundamental Theorem, we get

$$
N_{E}^{1)}\left(r, \frac{1}{\mathcal{F}-1}\right)=N_{E}^{1)}\left(r, \frac{1}{\mathcal{G}-1}\right) \leq N\left(r, \frac{1}{\mathcal{H}}\right) \leq N(r, \mathcal{H})+S(r, \mathcal{F})+S(r, \mathcal{G}) .
$$

Lemma 3. Let the set $\mathcal{S}_{2}$ be given as in Theorem 1 and $\Psi$ is given by (3). If $E_{f}\left(\mathcal{S}_{2}, n\right)=$ $E_{g}\left(\mathcal{S}_{2}, n\right)$ and $E_{f}\left(\mathcal{S}_{3}, n-2\right)=E_{g}\left(\mathcal{S}_{3}, n-2\right)$ and $\Psi \not \equiv 0$, then

$$
\bar{N}\left(r, \frac{1}{f}\right)+\bar{N}\left(r, \frac{1}{f-\delta_{a, b}^{n}}\right) \leq \bar{N}\left(r, \frac{1}{\mathcal{F}-1} \mid \geq n+1\right)+\bar{N}(r, f \mid \geq n-1)+S(r, f) .
$$

Proof. Since $\Psi \not \equiv 0$, so in view of the lemma of the logarithmic derivative, we have $m(r, \Psi)=$ $S(r, f)$. Again since $E_{f}\left(\mathcal{S}_{2}, n\right)=E_{g}\left(\mathcal{S}_{2}, n\right)$ and $E_{f}\left(\mathcal{S}_{3}, n-2\right)=E_{g}\left(\mathcal{S}_{3}, n-2\right)$, then one can note that

$$
N(r, \Psi) \leq \bar{N}\left(r, \frac{1}{\mathcal{F}-1} \mid \geq n+1\right)+\bar{N}(r, f \mid \geq n-1)+S(r, f) .
$$

Let $z_{0}$ be a point such that $f\left(z_{0}\right)=0$ or $f\left(z_{0}\right)=\delta_{a, b}^{n}$. Then since $E_{f}\left(\mathcal{S}_{1}, 0\right)=E_{g}\left(\mathcal{S}_{1}, 0\right)$, we must have $\Psi\left(z_{0}\right)=0$. Thus we see that

$$
\bar{N}\left(r, \frac{1}{f}\right)+\bar{N}\left(r, \frac{1}{f-\delta_{a, b}^{n}}\right) \leq N\left(r, \frac{1}{\Psi}\right) .
$$

Applying the First Fundamental Theorem, we get from (4) and (5),

$$
\begin{gathered}
\bar{N}\left(r, \frac{1}{f}\right)+\bar{N}\left(r, \frac{1}{f-\delta_{a, b}^{n}}\right) \leq N\left(r, \frac{1}{\Psi}\right) \leq \\
\leq T(r, \Psi)+S(r, f)=N(r, \Psi)+m(r, \Psi)+S(r, f)= \\
=N(r, \Psi)+S(r, f) \leq \bar{N}\left(r, \frac{1}{\mathcal{F}-1} \mid \geq n+1\right)+\bar{N}(r, f \mid \geq n-1)+S(r, f) .
\end{gathered}
$$


Lemma 4 ([6]). Let $a_{1}, a_{2}, a_{3}, a_{4}$ be four distinct complex numbers. If $E_{f}\left(a_{j}, \infty\right)=$ $E_{g}\left(a_{j}, \infty\right),(j=1,2,3,4)$, then $f(z)=\frac{\alpha g(z)+\beta}{\gamma g(z)+\delta}$, where $\alpha \delta-\beta \gamma \neq 0$.

Lemma $5([6])$. If $E_{f^{*}}(1, \infty)=E_{g^{*}}(1, \infty)$ with $\delta_{2}\left(0 ; f^{*}\right)+\delta_{2}\left(0 ; g^{*}\right)+\delta_{2}\left(\infty, f^{*}\right)+\delta_{2}\left(\infty, g^{*}\right)>$ 3 , then either $f^{*} g^{*} \equiv 1$ or $f^{*} \equiv g^{*}$.

\section{Proof of the theorem.}

Proof of Theorem 1. Let $\mathcal{F}$ and $\mathcal{G}$ be given by (1) and $\mathcal{H}$, by (2). We now discuss the following cases.

Case 1. Let $\mathcal{H} \not \equiv 0$. Therefore it is clear that $\mathcal{F} \not \equiv \mathcal{G}$ and hence $\Psi \not \equiv 0$. By the lemma of the logarithmic derivative, one can easily get that $m(r, \mathcal{H})=S(r, f)+S(r, g)=m(r, \Psi)$. Since $E_{f}\left(\mathcal{S}_{1}, 0\right)=E_{g}\left(\mathcal{S}_{1}, 0\right), E_{f}\left(\mathcal{S}_{2}, n\right)=E_{g}\left(\mathcal{S}_{2}, n\right)$ and $E_{f}\left(\mathcal{S}_{3}, n-2\right)=E_{g}\left(\mathcal{S}_{3}, n-2\right)$ from the construction of $\mathcal{H}$, one can easily get that

$$
\begin{gathered}
N(r, \mathcal{H}) \leq \bar{N}\left(r, \frac{1}{\mathcal{F}-1} \mid \geq n+1\right)+\bar{N}(r, f \mid \geq n-1)+\bar{N}\left(r, \frac{1}{f}\right)+\bar{N}\left(r, \frac{1}{f-\delta_{a, b}^{n}}\right)+ \\
+N_{0}\left(r, \frac{1}{f^{\prime}}\right)+N_{0}\left(r, \frac{1}{g^{\prime}}\right)+S(r, f)+S(r, g)
\end{gathered}
$$

where $N_{0}\left(r, \frac{1}{f^{\prime}}\right)$ denotes the counting function of those zeros of $f^{\prime}$ which are not the zeros of $f\left(f-\delta_{a, b}^{n}\right)(\mathcal{F}-1)$. Similarly, $N_{0}\left(r, \frac{1}{g^{\prime}}\right)$ can be defined.

By applying the Second Fundamental Theorem, we get

$$
\begin{aligned}
& (n+1)\{T(r, f)+T(r, g)\} \\
\leq & \bar{N}\left(r, \frac{1}{\mathcal{F}-1}\right)+\bar{N}(r, f)+\bar{N}\left(r, \frac{1}{f}\right)+\bar{N}\left(r, \frac{1}{f-\delta_{a, b}^{n}}\right)+\bar{N}\left(r, \frac{1}{\mathcal{G}-1}\right) \\
& +\bar{N}(r, g)+\bar{N}\left(r, \frac{1}{g}\right)+\bar{N}\left(r, \frac{1}{g-\delta_{a, b}^{n}}\right)-N_{0}\left(r, \frac{1}{f^{\prime}}\right)-N_{0}\left(r, \frac{1}{g^{\prime}}\right) \\
& +S(r, f)+S(r, g) .
\end{aligned}
$$

Now by using Lemmas 2, 3 and (6), we get from (7)

$$
\begin{gathered}
(n+1)\{T(r, f)+T(r, g)\} \leq \\
\leq \bar{N}\left(r, \frac{1}{\mathcal{F}-1} \mid \geq n+1\right)+N_{n-1}(r, f)+\bar{N}\left(r, \frac{1}{\mathcal{G}-1}\right)+\bar{N}\left(r, \frac{1}{\mathcal{F}-1} \mid \geq 2\right), \\
\bar{N}(r, g)+3\left\{\bar{N}(r, f \mid \geq n-1)+\bar{N}\left(r, \frac{1}{\mathcal{F}-1} \mid \geq n+1\right)\right\}+S(r, f)+S(r, g) \leq \\
\leq N_{n-1}(r, f)+N_{n-1}(r, g)+\frac{1}{n-1} N(r, f)+\frac{1}{n-1} N(r, g)+ \\
+\left\{2 \bar{N}\left(r, \frac{1}{\mathcal{F}-1} \mid \geq n+1\right)+\bar{N}\left(r, \frac{1}{\mathcal{F}-1} \mid \geq 2\right)\right\}+ \\
+\left\{\bar{N}\left(r, \frac{1}{\mathcal{G}-1}\right)+2 \bar{N}\left(r, \frac{1}{\mathcal{F}-1} \mid \geq n+1\right)\right\}+S(r, f)+S(r, g) .
\end{gathered}
$$


Next, we see that

$$
\begin{gathered}
\frac{1}{2} \bar{N}\left(r, \frac{1}{\mathcal{F}} \mid \leq 1\right)+\bar{N}\left(r, \frac{1}{\mathcal{F}} \mid \geq 2\right)+2 \bar{N}\left(r, \frac{1}{\mathcal{F}} \mid \geq n+1\right) \leq \\
\quad \leq \frac{1}{2} N\left(r, \frac{1}{\mathcal{F}-1}\right)+\bar{N}\left(r, \frac{1}{\mathcal{F}-1} \geq n+1\right) \leq \\
\leq\left(\frac{1}{2}+\frac{1}{n+1}\right) N\left(r, \frac{1}{\mathcal{F}-1}\right)=\frac{n+3}{2(n+1)} N\left(r, \frac{1}{\mathcal{F}-1}\right) .
\end{gathered}
$$

Similarly, we get

$$
\begin{aligned}
\frac{1}{2} \bar{N}\left(r, \frac{1}{\mathcal{G}} \mid \leq 1\right) & +\bar{N}\left(r, \frac{1}{\mathcal{G}} \mid \geq 2\right)+2 \bar{N}\left(r, \frac{1}{\mathcal{G}} \mid \geq n+1\right) \leq \\
& \leq \frac{n+3}{2(n+1)} N\left(r, \frac{1}{\mathcal{G}-1}\right) .
\end{aligned}
$$

Therefore, using (9) and (10), we obtain from (8)

$$
(n+1)\{T(r, f)+T(r, g)\} \leq\left(1+\frac{1}{n-1}+\frac{n(n+3)}{2(n+1)}\right)\{T(r, f)+T(r, g)\},
$$

which contradicts $n \geq 3$.

Case 2. Let $\mathcal{H} \equiv 0$. Then on integrating, we get from (2)

$$
\frac{1}{\mathcal{F}-1} \equiv \frac{\mathcal{A}}{\mathcal{G}-1}+\mathcal{B}, \text { where } \mathcal{A}(\neq 0), \mathcal{B} \in \mathbb{C} .
$$

From (11), we obtain in view Lemma 1 that

$$
T(r, f)=T(r, g)+S(r, f)+S(r, g) .
$$

Let $\infty$ be a Picard exceptional value of $f$. Then we must have $\bar{N}(r, f)=S(r, f)$.

From the proof of Lemma 3, we already have

$$
\begin{gathered}
\bar{N}\left(r, \frac{1}{f}\right)+\bar{N}\left(r, \frac{1}{f-\delta_{a, b}^{n}}\right) \leq \bar{N}\left(r, \frac{1}{\mathcal{F}-1} \mid \geq n+1\right)+\bar{N}(r, f \mid \geq n-1)+S(r, f) \leq \\
\leq \frac{1}{n+1} N\left(r, \frac{1}{\mathcal{F}-1}\right)+\frac{1}{n-1} \bar{N}(r, f)+S(r, f) \leq \frac{n}{n+1} T(r, f)+S(r, f)
\end{gathered}
$$

By the Second Fundamental Theorem and (12), we obtain

$$
T(r, f) \leq \bar{N}\left(r, \frac{1}{f}\right)+\bar{N}\left(r, \frac{1}{f-\delta_{a, b}^{n}}\right)+\bar{N}(r, f)+S(r, f) \leq \frac{n}{n+1} T(r, f)+S(r, f),
$$

which is a contradiction.

Suppose that $\infty$ is not a Picard exceptional value of $f$. So there must exits $z_{0} \in \mathbb{C}$ such that $f\left(z_{0}\right)=\infty$. Since $E_{f}\left(\mathcal{S}_{3}, n-2\right)=E_{g}\left(\mathcal{S}_{3}, n-2\right)$, we get from (11) that $\mathcal{B}=0$.

Therefore, we have $\mathcal{A}(\mathcal{F}-1) \equiv(\mathcal{G}-1)$, i.e.

$$
\mathcal{A}\left(a f^{n}+b f^{n-1}+c\right) \equiv\left(a g^{n}+b g^{n-1}+c\right) .
$$

Since $E_{f}\left(\mathcal{S}_{1}, 0\right)=E_{g}\left(\mathcal{S}_{1}, 0\right)$, we have the following two possibilities. 
(i) $E_{f}(0,0)=E_{g}(0,0)$ and $E_{f}\left(\delta_{a, b}^{n}, 0\right)=E_{g}\left(\delta_{a, b}^{n}, 0\right)$, or

(ii) $E_{f}(0,0)=E_{g}\left(\delta_{a, b}^{n}, 0\right)$ and $E_{f}\left(\delta_{a, b}^{n}, 0\right)=E_{g}(0,0)$.

Subcase 2.1. Suppose $E_{f}(0,0)=E_{g}(0,0)$ and $E_{f}\left(\delta_{a, b}^{n}, 0\right)=E_{g}\left(\delta_{a, b}^{n}, 0\right)$. Then there exist $z_{0}, z_{1} \in \mathbb{C}$ such that $f\left(z_{0}\right)=0=g\left(z_{0}\right)$ and $f\left(z_{1}\right)=\delta_{a, b}^{n}=g\left(z_{1}\right)$. In both the cases, we get from (13) that $\mathcal{A}=1$. Then (13) reduces to

$$
a f^{n}+b f^{n-1} \equiv a g^{n}+b g^{n-1} \text {. i.e., } f^{n-1}(a f+b) \equiv g^{n-1}(a g+b)
$$

Since $E_{f}(0,0)=E_{g}(0,0)$, from $(14)$, we get $E_{f}\left(-\frac{b}{a}, 0\right)=E_{g}\left(-\frac{b}{a}, 0\right)$. Again since $E_{f}\left(\mathcal{S}_{3}, n-2\right)=E_{g}\left(\mathcal{S}_{3}, n-2\right)$, we see that

$$
\begin{gathered}
E_{f}(0,0)=E_{g}(0,0), \quad E_{f}\left(\delta_{a, b}^{n}, 0\right)=E_{g}\left(\delta_{a, b}^{n}, 0\right), \\
E_{f}\left(-\frac{b}{a}, 0\right)=E_{g}\left(-\frac{b}{a}, 0\right), \quad E_{f}(\infty, n-2)=E_{g}(\infty, n-2) .
\end{gathered}
$$

Then by Lemma 4, one must have

$$
f(z)=\frac{\alpha g(z)+\beta}{\gamma g(z)+\delta}
$$

where $\alpha \delta-\beta \gamma \neq 0$. Therefore, equations (14) and (15) combinedly give $f \equiv g$.

Subcase 2.2. Suppose $E_{f}(0,0)=E_{g}\left(\delta_{a, b}^{n}, 0\right)$ and $E_{f}\left(\delta_{a, b}^{n}, 0\right)=E_{g}(0,0)$.

We now discuss the following subcases.

Subcase 2.2.1. Let both $E_{f}(0,0)=E_{g}\left(\delta_{a, b}^{n}, 0\right)=\varnothing$ and $E_{f}\left(\delta_{a, b}^{n}, 0\right)=E_{g}(0,0)=\varnothing$. Since $E_{f}(\infty, n-2)=E_{g}(\infty, n-2)$, so we must have $E_{f^{*}}(1, n-2)=E_{g^{*}}(1, n-2)$, where $f^{*}(z)=$ $\frac{f(z)}{f(z)-\delta_{a, b}^{n}} \neq 0, \infty$ and $g^{*}(z)=\frac{g(z)-\delta_{a, b}^{n}}{g(z)} \neq 0, \infty$. Again we note that

$$
\delta_{2}\left(0 ; f^{*}\right)+\delta_{2}\left(0 ; g^{*}\right)+\delta_{2}\left(\infty, f^{*}\right)+\delta_{2}\left(\infty, g^{*}\right)=4>3 .
$$

Therefore, by using Lemma 5 , we have $f^{*} \equiv g^{*}$ or $f^{*} g^{*} \equiv 1$.

Subcase 2.2.1.1. Suppose $f^{*} g^{*} \equiv 1$. Then we have $f \equiv g$.

Subcase 2.2.1.2. Suppose $f^{*} \equiv g^{*}$. Then we have

$$
f+g=\delta_{a, b}^{n} .
$$

Thus from (13) and (16), we see that $f$ is a constant, which is absurd.

Subcase 2.2.2. Let $E_{f}(0,0)=E_{g}\left(\delta_{a, b}^{n}, 0\right)=\varnothing$ or $E_{f}\left(\delta_{a, b}^{n}, 0\right)=E_{g}(0,0)=\varnothing$.

Subcase 2.2.2.1. Suppose $E_{f}(0,0)=E_{g}\left(\delta_{a, b}^{n}, 0\right)=\varnothing$ and $E_{f}\left(\delta_{a, b}^{n}, 0\right)=E_{g}(0,0) \neq \varnothing$. This implies that there exists $z_{0} \in \mathbb{C}$ such that $f\left(z_{0}\right)=\delta_{a, b}^{n}$ and $g\left(z_{0}\right)=0$. So from (13), we get

$$
\mathcal{A}=\frac{a\left(\delta_{a, b}^{n}\right)^{n}+b\left(\delta_{a, b}^{n}\right)^{n-1}+c}{c} .
$$


It follows from (17) that

$$
-a\left(\delta_{a, b}^{n}\right)^{n}-b\left(\delta_{a, b}^{n}\right)=c(1-\mathcal{A}) .
$$

Clearly, one root of the equation (18) is $\delta_{a, b}^{n}$ of multiplicity 2. Equation (13) can be written as

$$
a f^{n}+b f^{n-1}+c-\frac{c}{\mathcal{A}}=\frac{1}{\mathcal{A}}\left(a g^{n}+b g^{n-1}\right) .
$$

We must have $c-\frac{c}{\mathcal{A}} \neq c(1-\mathcal{A})$, otherwise we will have $\mathcal{A}= \pm 1$, which is a contradiction as $c \neq-\frac{b}{2 n}\left(\delta_{a, b}^{n}\right)^{n-1}, \delta_{a, b}^{n} \neq-\frac{b}{a}, 0$.

Now, equation (19) can be written as

$$
a \prod_{j=1}^{n}\left(f-\zeta_{j}\right)=\frac{1}{\mathcal{A}} g^{n-1}(a g+b)
$$

where $\zeta_{j}(j=1,2, \ldots, n)$ are distinct roots of the polynomial $a f^{n}+b f^{n-1}+c-\frac{c}{\mathcal{A}}$. From (20), it is clear that 0 is a Picard exceptiona value of $g$, which contradicts our assumption $E_{f}\left(\delta_{a, b}^{n}, 0\right)=E_{g}(0,0) \neq \varnothing$.

Subcase 2.2.2.2. Suppose $E_{f}(0,0)=E_{g}\left(\delta_{a, b}^{n}, 0\right) \neq \varnothing$ and $E_{f}\left(\delta_{a, b}^{n}, 0\right)=E_{g}(0,0)=\varnothing$. This implies that there exists $z_{1} \in \mathbb{C}$, such that $f\left(z_{1}\right)=0$ and $g\left(z_{1}\right)=\delta_{a, b}^{n}$. Then from (13), we get

$$
\mathcal{A}=\frac{c}{a\left(\delta_{a, b}^{n}\right)^{n}+b\left(\delta_{a, b}^{n}\right)^{n-1}+c} .
$$

Next proceeding exactly same way as done in Subcase 2.2.2.1, we get a contradiction.

Subcase 2.2.3. Suppose both $E_{f}(0,0)=E_{g}\left(\delta_{a, b}^{n}, 0\right) \neq \varnothing$ and $E_{f}\left(\delta_{a, b}^{n}, 0\right)=E_{g}(0,0) \neq \varnothing$. Then we get

$$
\mathcal{A}=\frac{a\left(\delta_{a, b}^{n}\right)^{n}+b\left(\delta_{a, b}^{n}\right)^{n-1}+c}{c} \text { and } \mathcal{A}=\frac{c}{a\left(\delta_{a, b}^{n}\right)^{n}+b\left(\delta_{a, b}^{n}\right)^{n-1}+c} .
$$

Thus we see that $\mathcal{A}= \pm 1$, which contradicts $c \neq-\frac{b}{2 n}\left(\delta_{a, b}^{n}\right)^{n-1}$.

4. Concluding remarks and a question. In this paper, we proved a result with the best possible cardinalities of the three sets sharing problems till now by answering the question posed by Yi ([17]) without the help of any extra suppositions. We have also abled to relax the nature of sharing of the sets compare to other results mentioned in the introduction. But we do not know whether the choice of the weights $\left(k_{1}, k_{2}, k_{3}\right)=(0,3,1)$ associated with the corresponding sets $\mathcal{S}_{j}, j=1,2,3$, in our main result is the best possible or not. So we have the following quarry for the future investigation in this direction.

Question 5. Keeping all other conditions intact in Theorem 1, is it possible to relax the nature of sharing of the sets further?

Acknowledgment. The author would like to thank the referee for his/her helpful suggestions and comments towards the improvement of this manuscript. 


\section{REFERENCES}

1. Banerjee A., Some uniqueness results on meromorphic functions sharing three sets, Ann. Polon. Math., 92 (2007), №3, 261-274.

2. Banerjee A., Uniqueness of meromorphic functions that share three sets, Kyungpook Math. J., 49 (2009), $15-29$.

3. Banerjee A., Ahamed M.B., On uniqueness of meromorphic functions sharing three sets with finite weights, Bull. Polish Acad. Sci., 62 (2014), №3, 243-256.

4. Banerjee A., Majumder S., Uniqueness of meromorphic functions sharing three sets-further study, Analysis, 34 (2014), №2, 143-162.

5. Banerjee A., Mukherjee S., Uniqueness of meromorphic functions sharing two or three sets, Hokkaido Math. J., 37 (2008), 507-530.

6. Fang M.L., Uniqueness of admissible meromorphic functions in the unit disc, Sci. China (A), 42 (1999), №4, 367-381.

7. Fang M., Xu W., A note on a problem of Gross, Chinese J. Contemporary Math., 18 (1997), №4, 395-402.

8. Gross F., Factorization of meromorphic functions and some open problems, Proc. Conf. Univ. Kentucky, Leixngton, Ky(1976); Complex Analysis, Lecture Notes in Math., 599 (1977), 51-69, Springer Verlag.

9. Hayman W.K., Meromorphic Functions, The Clarendon Press, Oxford, 1964.

10. Lahiri I., Weighted sharing and uniqueness of meromorphic functions, Nagoya Math. J., 161 (2001), 193-206.

11. Lahiri I., Weighted value sharing and uniqueness of meromorphic functions, Complex Var. Theory Appl., 46 (2001), 241-253.

12. Lahiri I., Sarkar A., Uniqueness of meromorphic function and its derivative, J. Inequal. Pure Appl. Math., 5 (2004), №1, Art.20.

13. Lin W.C., Yi H.X., Uniqueness theorems for meromorphic functions that share three sets, Complex Var. Theory Appl., 48 (2003), 315-327.

14. Mokhonko A.Z., On the Nevanlinna characteristics of some meromorphic functions, Theo. Funct. Funct. Anal. Appl., Izd-vo Kharkovsk Un-ta, 14 (1971), 83-87.

15. Qiu H., Fang M., A unicity theorem for meromorphic functions, Bull. Malaysian Math. Sci. Soc., 25 (2002), 31-38.

16. Yi H.X., On characteristic function of a meromorphic function and its derivative, Indian J. Math., 33 (1991), №2, 119-133.

17. Yi H.X., Uniqueness of meromorphic functions and a question of Gross, Science in China, 37 (1994), №7A, 802-813.

18. Yi H.X., Lin W.C., Uniqueness theorems concerning a question of Gross, Proc. Japan Acad. Ser. A, 80 (2004), 136-140.

19. Zhang Q.C., Meromorphic function that shares one small function with its derivative, J. Inequal. Pure Appl. Math., 6 (2005), №4, Art.116.

Department of Mathematics, Kalipada Ghosh Tarai Mahavidyalya

West Bengal, India

basir_math_kgtm@yahoo.com

bsrhmd117@gmail.com

Received 3.05.2019

Revised 2.05.2020 